\title{
Multiscale Denoising and Enhancement of 3D Rotational X-ray Imaging for Percutaneous Vertebroplasty
}

\author{
Yinpeng Jin ${ }^{1}$, Elsa Angelini ${ }^{1}$, Sundeep Mangla ${ }^{2,3}$, In Sup Choi ${ }^{4}$, Richard Kemkers ${ }^{5}$, Jan Timmer ${ }^{6}$, Andrew Laine ${ }^{1}$ \\ ${ }^{1}$ Department of Biomedical Engineering, Columbia University, New York, NY, USA \\ ${ }^{2}$ Department of Radiology, New York Presbyterian Hospital, New York, NY, USA \\ ${ }^{3}$ Division of Interventional Neuroradiology, SUNY-Downstate Medical Center, Brooklyn, NY, USA \\ ${ }^{4}$ Lahey Medial Center, Burlington, MA, USA \\ ${ }^{5}$ Philips Medical Systems North America, Bothell, WA, USA \\ ${ }^{6}$ Philips Medical Systems Nederland BV, Best, the Netherlands
}

\begin{abstract}
D rotational X-ray (3DRX) imaging has been suggested as an alternative to $C T$ scans for evaluating percutaneous vertebroplasty procedures. We present in this study preliminary work on denoising and enhancement of 3DRX data sets with dyadic wavelet thresholding. Thresholding operators were tuned to accommodate for spatial variability of the anatomical features and the noise energy at each level of decomposition. Results are presented for three clinical data sets with quantitative measurements of contrast to noise ratio (CNR) and qualitative evaluation with threedimensional rendering of vertebral bodies having cement fillings. Wavelet denoising demonstrated the efficient on enhancement of subtle vertebral edges and clearer delineation of the cement filling contours which improves the accuracy in clinical practice and helps to make 3DRX more competitive when compared to CT.
\end{abstract}

Keywords - 3D Rotational X-ray, CNR, Enhancement, Percutaneous Vertebroplasty, Wavelet thresholding.

\section{INTRODUCTION}

Since the introduction of percutaneous vertebroplasty in the United States in 1993, this procedure has been mainly applied to patients suffering from osteoporosis [1]. The incidence of osteoporosis in the United States is high in terms of hospital care with 700,000 osteoporotic vertebral compression fractures per year, leading to 115,000 hospital admissions. Vertebroplasty has proved to be an effective method that aims at stabilizing fractures in the spinal column [2].

The vertebroplasty procedure is generally performed under fluoroscopic control for proper needle insertion and localization of poly-methylmethacrylate (PMMA) cement filling. A post-procedural CT scan is sometimes used to monitor the distribution of PMMA cement, requiring that a CT scanner is available in the vicinity of the interventional angio-room. This, however, adds radiation exposure to the global procedure. The recent introduction of 3D rotational X-ray (3DRX) imaging offers an excellent alternative to CT scans with the advantage of immediate post-assessment capabilities using the same X-ray equipment as used for injection procedure, with relatively lower dosage of radiation.

However, clinical acceptance of the use of 3DRX images for post-procedure evaluation requires an improvement in image quality with respect to precise boundary definition and lower noise levels which are essential for accurately identifying the following important clinically significant findings:

1.Distribution of PMMA cement: if outside the vertebral body, this could bring a certain level of danger depending on the location (spinal canal, parvertebral veins, etc.)

2.Degree (percentage) of total filling within the vertebral body: may determine whether or not the procedure ultimately provides adequate symptom relief or stability of spine.

3. Intactness of vertebral body: evaluates the continuity of the margins of vertebral body and fracture lines, and identifies the presence of the bone fragments within the canal.

These pieces of information are derived from visual inspection of the data. Image quality will therefore directly influence the quality and accuracy of the post-intervention evaluation via rendering of the vertebral bone structures. This requires high contrast and signal-to-noise ratio (SNR) at vertebral edges and cement graft locations.

Many patients undergoing vertebroplasty have low bone density (i.e. osteopenic). In this case, bone structure shows similar pixel intensity to the background noise, thus leading to low contrasted X-Ray images.

In all cases, particularly with osteopenic patients, elimination of the noise component and increase of the local contrast at bone to soft tissue interfaces are useful to guarantee the safety and efficacy of a vertebroplasty procedure.

Multi-scale analysis using wavelet analysis has been shown to offer a very flexible and powerful mathematical framework for denoising and enhancement of highresolution medical images with comparable or even superior performance when compared to traditional methods such as filtering [3-7]. In this study, we developed a linear spatial adaptive paradigm targeting simultaneous denoising and enhancement of 3DRX volume data sets from postvertebroplasty scanning. We focus on both qualitative and quantitative improvement of the visualization of landmark anatomical structures.

\section{METHODOLOGY}

\section{A. Three Dimensional Dyadic Wavelet Expansion}

Unlike a discrete orthogonal wavelet transform, we explore an overcomplete representation of a dyadic wavelet 
expansion which provides translation invariance [8]. We implemented the wavelet transform in 3D to fully exploit the volumetric information recorded by the $3 \mathrm{DRX}$ scanner. The first derivative of a cubic spline function was used for the multi-resolution expansion. Such a wavelet transform isolates preeminent gradient information in the transform domain so that wavelet coefficients with larger magnitude are correlated with edge locations in a three dimensional space.

Denoising with wavelet thresholding is achieved by attenuating wavelet coefficients that represent mostly noise components. Enhancement, on the other hand, is achieved by amplifying wavelet coefficients that were identified as part of an object's boundary or points (features) of interest. The denoised/enhanced signal is then reconstructed from the modified wavelet coefficients.

In the present study, at each level of analysis, a 3D dyadic wavelet expansion produced coefficients with three components analogous to the Cartesian components of a gradient vector. Previous work performed by our group using the 3D dyadic wavelet expansion [9] suggested that thresholding on modulus of the wavelet coefficients, computed as the magnitude of the coefficient vector, instead of the individual coefficient components provided extra selectivity on the directional information and lead to more robust results for denoising and enhancement on three or higher dimensional data [10]. In this case, the modulus of wavelet coefficients was thresholded but the phase information was preserved for reconstruction.

\section{B. Multiscale Denoising with Spatial Adaptive Thresholding}

With the assumption that larger valued wavelet coefficients correlate to edge information while smaller values represent noise variations, multi-scale denoising was implemented via soft thresholding which resets small-value wavelet coefficients to zero and reduces remaining coefficients towards zero according to threshold levels [3].

Image data, especially medical images, typically display strong inhomogeneities across individual anatomical features. With this type of data, global thresholding strategies that cannot adapt to such local context or data information have been found to be suboptimal in terms of efficiency and robustness for denoising [11]. With this in mind, spatial adaptive thresholding strategies have recently been suggested by several groups $[11,12]$ to improve the denoising performance on images that are not characterized by "piecewise smooth" representations.

Further analysis of wavelet coefficients distributions often shows that regions with higher variance usually correspond to significant features such as edges and textures, while lower variance regions are often dominated by noise [11]. This suggests that an optimal threshold level should be inversely proportional to the local signal variance $\sigma_{X}$ estimated in the wavelet transform domain. Such a strategy preserves coefficients for regions with significant features (with higher $\sigma_{X}$ ), while thresholding aggressively in smooth regions dominated by noise (with lower $\sigma_{X}$ ).

In this study, we tested a soft thresholding operator initially proposed by Donoho [3], setting the normalized threshold value to $T=c / \sigma_{X}$, where $c$ is a user defined constant which depends on the level of analysis (i.e. scale) in the transform domain. We point out here that an automatic selection of $c$ was proposed in [11] based on an estimation of the noise standard deviation $\sigma_{n}$.

\section{Spatial Adaptive Edge Enhancement}

Considering that edge definition is essential for 3DRX data in vertebroplasty, we further extended the adaptivity of the threshold level to accommodate for spatial variation of the anatomical features and included an edge enhancement operator. We previously tested a similar thresholding approach for SPECT brain data [13] following a design suggested by Chang et al. in [11]. In this work we approached the spatial adaptivity of the threshold level by identifying, in the noisy data, edge locations with a Canny detector and applying enhancement at their locations after thresholding of the coefficients. With this approach, denoising is performed first by decreasing the coefficients amplitude while enhancement is achieved by adaptively increasing the coefficients magnitude. Because of the overcomplete structure of our wavelet implementation, this methodology could be applied in a straightforward manner with a direct spatial correspondence of the data and its representation in the transform domain.

\section{RESULTS}

\section{A. 3DRX Acquisition}

Three-dimensional digital rotational radiography was performed using a protocol of 180 degree fan rotation centered at the vertebral body of interest. Data acquisition was performed using a $512 \times 512$ image matrix at the preferred field of view. These images were obtained using a Philips Integris Biplane Neurovascular System with appropriate $\mathrm{mAs}$ and $\mathrm{kV}$ required for radiographic acquisition as determined by each individual patient's body habitus and automated dose adjustment.

\section{B. Denoising Results on Clinical Data}

Denoising was performed on three clinical data sets with a two-level dyadic wavelet transform and soft thresholding of the expansion coefficients prior to reconstruction. The thresholding level coefficient $c$ was manually set for each level in the range [ $\left[\begin{array}{ll}0 & 0.2\end{array}\right]$ while the standard deviation $\sigma_{X}$ was estimated in a local sub-volume with size of $7 \times 7 \times 7$ for each pixel. For the spatial adaptive thresholding, an edge map of the original data was computed using a Canny detector to define locations of the edges to enhance after thresholding for denoising. The 
threshold level coefficient $e$ for enhancement was again set manually at each level in the range [1.0 2.0].

For comparison purposes, Gaussian filtering with standard deviation $\sigma$ set to 1 was performed in $3 \mathrm{D}$. An illustration of the denoising performance with each method on a single axial view is provided in Figure 2 for one clinical case.

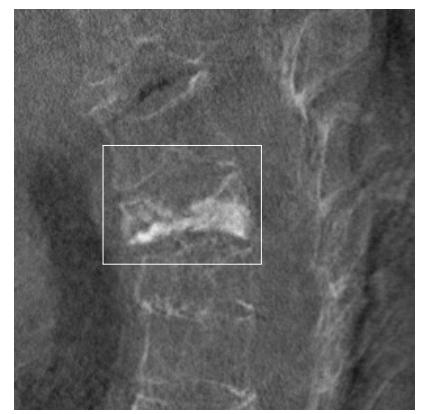

(a)

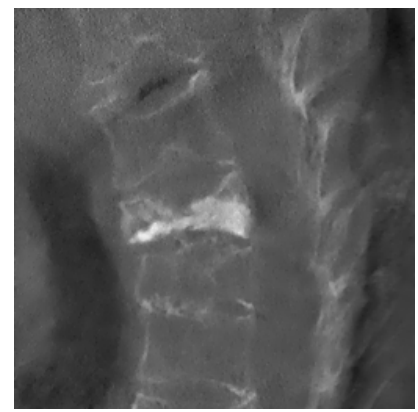

(c)

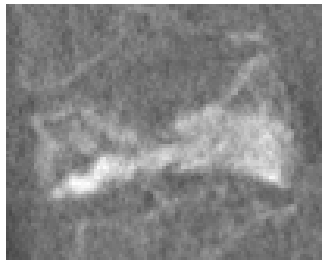

(e)

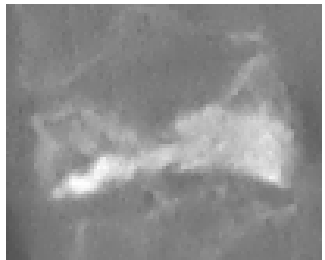

(g)

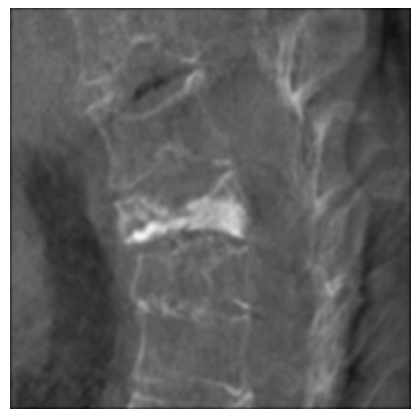

(b)

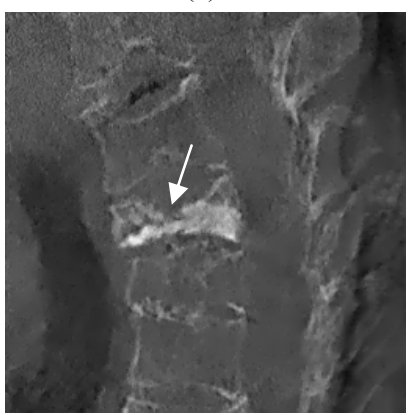

(d)

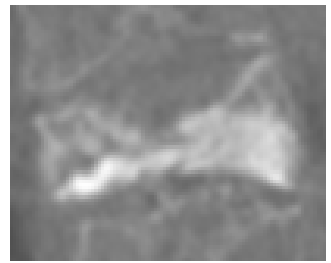

(f)

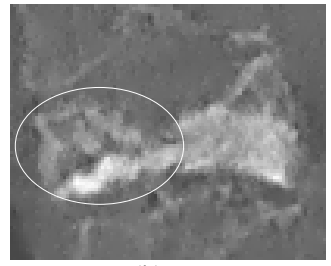

(h)
Figure 1: (a) Original 3DRX data. Denoised image by: (b) Gaussian filtering, (c) wavelet shrinkage, (d) the proposed adaptive thresholding method. (e) (h) Magnified view (2×) of (a) (d) for the region of interest marked in (a). Notice the locations marked by arrow in (d) and oval in (h) show improvements of clinical importance.

Denoising performance was evaluated quantitatively with measurements of contrast to noise ratio (CNR), based on the definition used in [14]. Since the most useful information for vertebroplasty is the clear definition (contrast) between vertebral and surrounding tissues and between the filling cement and the bone structures, we measured CNR at selected vertebral-muscle and cement- bone interfaces with distinct degrees of contrast by manually defining "object" and "background" regions required for computing CNR. Sample measurements at selected locations were performed on three cases and are reported in Table 1.

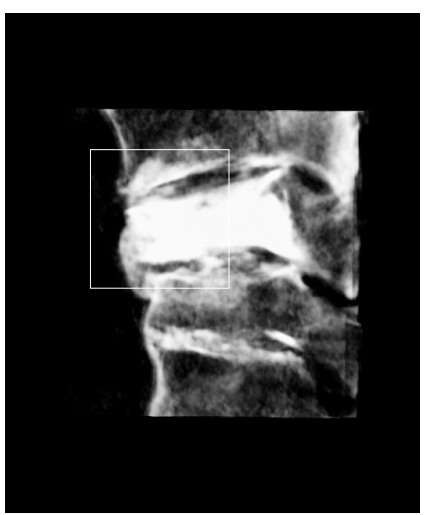

(a)

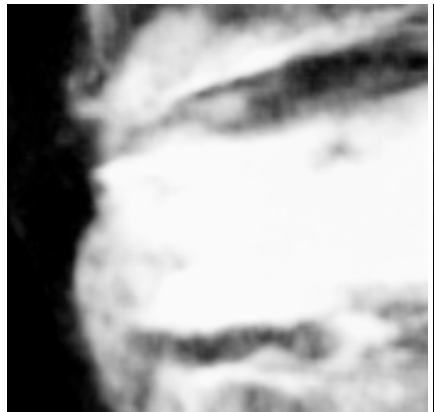

(c)

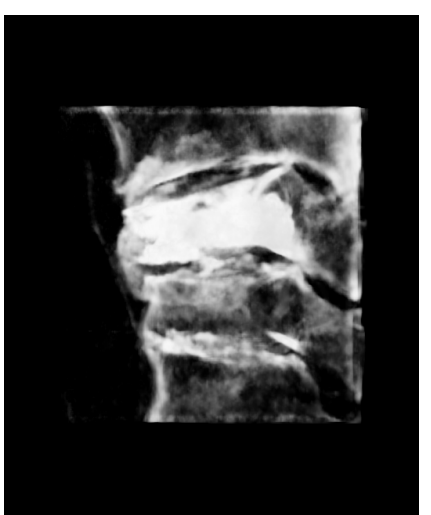

(b)

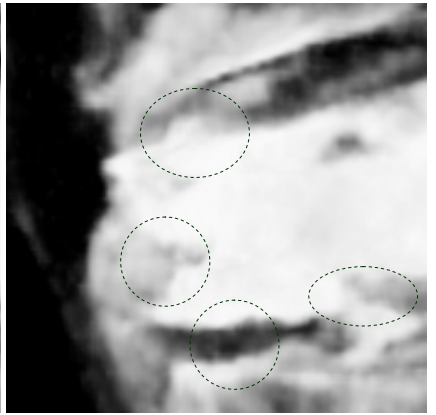

(d)
Figure 2: Volume rendering of: (a) original data, (b) denoised data by the proposed method. (c) (d) Magnified views $(3 \times)$ of $(a) \sim(b)$ for the region of interest marked in (a). Regions marked by dashed ovals in (d) show improvements of clinical importance.

We observed from the CNR measurements that the proposed denoising/enhancement method shows consistently better performance compared to two of the most commonly used denoising methods: Gaussian filtering and wavelet shrinkage. We claim that both denoising and edge enhancement contributed to the improvement of CNR measurement.

Table 1: Sample measurements of CNR (dB) at locations of bone-soft tissue interface (B-S) and cement-bone tissue interface (C-B) for the three testing cases Processed with Gaussian Filtering (GF), : Wavelet Shrinkage(WS), and Adaptive Thresholding (AT) which is the proposed method.

\begin{tabular}{|c|c|c|c|c|c|}
\hline \multicolumn{2}{|c|}{} & Original & GF & WS & AT \\
\hline Case 1 & B-S & 9.13 & 7.19 & 8.90 & 10.06 \\
\cline { 2 - 6 } & C-B & \multicolumn{4}{|c|}{ N/A } \\
\hline Case 2 & B-S & 8.39 & 7.28 & 8.51 & 8.67 \\
\cline { 2 - 6 } & C-B & 9.55 & 9.35 & 10.12 & 10.20 \\
\hline Case 3 & B-S & 6.61 & 3.45 & 4.95 & 6.66 \\
\cline { 2 - 6 } & C-B & 8.91 & 8.43 & 8.92 & 9.04 \\
\hline
\end{tabular}

Qualitative evaluation of the denoising method was performed by displaying $3 \mathrm{D}$ volume rendering of the data using a Philips clinical display console. Rendering for one 
case is displayed in Figure 2 at the location of the PMMA cement.

One of the critical clinical applications in 3D rendering of the 3D X-ray data set is the accurate localization of the cement filling inside the vertebral body. We observed a clear improvement of the data quality with the adaptive thresholding when compared to the original data, as demonstrated in Figure 2, where the original data grossly overestimated the extent of cement deposition (dashed ovals). The edges of PMMA cement were better defined with cleaner borders and improved shape delineation. This result lead to better appreciation of cement filling location and distribution as shown in Figure 1 (regions marked with arrow and oval).

\section{DISCUSSION}

Our quantitative and qualitative analysis shows that the adaptive soft thresholding of WT coefficients is very effective to enhance subtle edges of the vertebral bodies and to refine the appearance of cement distributions. Although statistical significance can not be claimed given the limited number of cases, we observed consistently superior enhancement at the locations of measurement when compared with two of the commonly used denoising methods: Gaussian filtering and wavelet shrinkage.

Due to the lack of ground truth data, measurements of CNR could only be carried out by manually tracing the "true" contours of the objects of interest (i.e. vertebral body and cement filing). With this method, we therefore needed to identify regions where the interface between the objects of interest could be accurately defined. Indeed the accuracy of the contours will affect directly the confidence of the CNR measurement. For this reason, no CNR measurement was performed on the (C-B) interface in Case 1 as no reliable edges could be drawn for the cement's contours. This result suggests that more robust quantitative measurement should be investigated to evaluate the performance of the denoising and enhancement on the clinical cases.

Regarding the algorithm, denoising and enhancement parameters were selected experimentally in this preliminary study. We point out that an automatic selection should be possible and will be investigated, with careful analysis of the statistics of the wavelet coefficients.

\section{CONCLUSION}

We have presented in this study initial results of denoising and enhancement of 3DRX volumes for vertebroplasty applications.

Full evaluation of the proposed denoising/enhancement method will involve a phantom study for measurements of SNR and assessment of spatial resolution improvement. Selection of a suitable phantom requires further investigation due to the novelty of the modality. It is also planned to compare the performance of the 3DRX modality to standard CT data. The CT modality is sometimes used to evaluate a vertebroplasty procedure despite drawbacks that include patient discomfort, CT scan times, and a need of a $\mathrm{CT}$ scanner in the proximity of the operating room. On the other hand 3DRX offers an excellent alternative with immediate post-assessment capabilities, increased spatial resolution with sufficient contrast resolution, as well as a single acquisition with lower radiation dosage.

\section{ACKNOWLEDGMENTS}

The authors would like to thank R\&D scientist Paul Desmedt from Philips Medical Systems Nederland BV, service engineer Paul DiRosario of Philips Medical Systems North America and X-ray technologist Stephen Knight from Lahey Clinical Medical Center (Burlington, MA, USA) for their respective efforts on creating the vertebroplasty prototype software, performing calibration, collecting data for this research and determining the acquisition protocols.

\section{REFERENCES}

[1] M. E. Jensen and J. E. Dion, "Verterbroplasty Relieves Osteoporosis Pain.," Diagn. Imaging, vol. 19, pp. 68-72, 1997.

[2] J. E. Dion, "Percutaneous Vertebroplasty," MEDICA MUNDI, vol. 45, pp. 20-28, 2001.

[3] D. L. Donoho, "De-noising by soft-thresholding," IEEE Transactions on Information Theory, vol. 41, pp. 613-627, 1995.

[4] A. F. Laine, S. Schuler, J. Fan, and W. Huda, "Mammographic feature enhancement by multiscale analysis," IEEE Transactions on Medical Imaging, vol. 13, pp. 725-740, 1994.

[5] R. Coifman and D. Donoho, "Translation invariant denoising," in Wavelet and Statistics: Springer-Verlag Lecture Notes, 1995.

[6] S. Mallat and S. Zhong, "Characterization of signals from multiscale edges," IEEE Transactions on Pattern Analysis and Machine Intelligence, vol. 14, pp. 710-732, 1992.

[7] A. Aldroubi and M. Unser, Wavelets in Medicine and Biology. Boca Raton, FL: CRC, 1996.

[8] I. Koren and A. Laine, "A discrete dyadic wavelet transform for multidimensional feature analysis," in Time Frequency and Wavelets in Biomedical Signal Processing, IEEE Press series in biomedical engineering, M. Akay, Ed. Piscataway, NJ: IEEE Press, 1998, pp. 425-448.

[9] S. Mallat, A Wavelet Tour of Signal Processing. San Diego, CA: Academic Press, 1998.

[10] J. Kalifa, A. Laine, and P. Esser, "Regularization in tomographic reconstruction using thresholding estimators," SPIE Conference on Wavelet applications, San Diego, vol. 4478, pp. 63-74, 2001.

[11] S. Chang, B. Yu, and M. Vetterli, "Spatially adaptive wavelet thresholding with context modeling for image denoising," IEEE Transactions on Image Processing, vol. 9, pp. 1522-1531, 2000.

[12] M. K. Mihcak, I. Kozintsev, and K. Ramchandran, "Spatially adaptive statistical modeling of wavelet image coefficients and its application to denoising," IEEE International Conference on Acoustic, Speech and Signal processing, vol. 6, pp. 3253-3256., 1999.

[13] E. D. Angelini, J. Kalifa, and A. F. Laine, "Harmonic multiresolution estimators for denoising and regularization of SPECT-PET data," International Symposium on Biomedical Imaging, Washington, D.C., USA, pp. 697-700, 2002.

[14] E. Meijering, W. Niessen, J. Weickert, and M. Viergever, "Diffusion-Enhanced Visualization and Quantification of Vascular Anomalies in Three-Dimensional Rotational Angiography: Results of an In-Vitro Evaluation," Medical Image Analysis, vol. 6, pp. 217-235, 2002. 